\section{Polymer brush patterning using self-assembled microsphere monolayers as microcontact printing stamps $\uparrow$}

\author{
Tao Chen, $\ddagger^{* a b}$ Rainer Jordan ${ }^{a}$ and Stefan Zauscher $* b$ \\ Received 18th March 2011, Accepted 4th May 2011 \\ DOI: 10.1039/c1sm05474k
}

\begin{abstract}
Self-assembled microsphere monolayers (SMMs) hold significant promise for micro- and nanopatterning. Here we exploit, for the first time, SMMs as stamps for microcontact printing $(\mu \mathrm{CP})$ and demonstrate this to fabricate patterned initiator templates that can subsequently be amplified into polymer brushes by surface initiated atom transfer radical polymerization (SI-ATRP). SMM stamps avoid the need for expensive and sophisticated instrumentation in pattern generation, and provide a broad range of accessible surface chemistries and pitch size control.
\end{abstract}

Monodisperse microspheres can self-assemble on solid substrates into 2D close-packed hexagonal arrays due to capillary forces that arise from the evaporation of the suspension medium. ${ }^{1,2}$ These selfassembled microsphere monolayers (SMMs) have been used in the past to fabricate hierarchical structures, ${ }^{3}$ Janus particles, ${ }^{4}$ and colloid crystals. ${ }^{5}$ Furthermore, Whitesides et al. used polystyrene (PS) SMMs as a master for the preparation of PDMS or PDMS/PS SMM stamps for microcontact printing $(\mu \mathrm{CP}) .{ }^{6}$ Microcontact printing, developed in the early 1990s for the patterned transfer of thiols from stamps onto Au surfaces, ${ }^{7}$ has since become a simple, versatile, and costeffective patterning approach for micro/nanofabrication. ${ }^{8-11}$ In this field, patterning of polymer brushes is a rapidly developing direction, ${ }^{12-14}$ because patterned polymer brush microstructures are potentially useful for array-based diagnostic platforms and for the study of stimuli-responsive phenomena. ${ }^{15}$ While early examples of fabricating patterned polymer brush microstructures by $\mu \mathrm{CP}$ involved printing an octadecyltrichlorosilane (OTS) pattern to direct the backfilling of the interspaces with an initiator, ${ }^{16,17}$ more recent, alternative approaches printed polymerization initiators directly. ${ }^{18-20}$

Here we exploit supported self-assembled microsphere monolayers as an innovative tool for directly printing patterned initiator templates that can be subsequently amplified into polymer brushes by

${ }^{a}$ Department of Chemistry, Technische Universität Dresden, Dresden, 01069, Germany.E-mail: tao.chen@chemie.tu-dresden.de

${ }^{b}$ Center for Biologically Inspired Materials and Materials Systems, and Department of Mechanical Engineering and Materials Science, Duke University,Durham, NC,27708,USA. E-mail: zauscher@duke.edu $\dagger$ Electronic supplementary information (ESI) available: Detailed methods and characterization. See DOI: 10.1039/c1sm05474k

\$ Previous address: Center for Biologically Inspired Materials and Materials Systems, and Department of Mechanical Engineering and Materials Science, Duke University, Durham, NC, 27708, USA. surface initiated atom transfer radical polymerization (SI-ATRP). Although SMMs served as masters for replica mold polymeric stamps, to our knowledge, SMMs have not previously been used as stamps for directly patterning substrate surfaces. ${ }^{2}$ Our work not only develops the concept of using SMMs as $\mu \mathrm{CP}$ stamps but also intimates that control over polymer brush morphologies can be achieved. For example, the surface chemical properties, and the feature and pitch size of a SMM stamp are controllable by choosing microspheres with different chemical functionalities and diameters, and ink transfer conditions can be manipulated over a range of printing loads and printing times.

Our patterning and fabrication strategy is schematically shown in Fig. 1. In the first step, a SMM is assembled onto a silicon wafer using a suitable suspension medium (Fig. 1A). Once the liquid phase has evaporated, the dry SMM stamp is inked with thiol initiator, and prior to printing, dried with nitrogen (Fig. 1B). Upon contact between the SMM stamp and a gold substrate, the ink is transferred onto the gold surface. The curvature of the spheres induces a radially symmetric initiator concentration gradient at each patterned spot. The initiator density is higher in the center than at the periphery due to ink diffusion (Fig. 1C). This initiator gradient pattern can subsequently be amplified into cone-shaped brush microstructures via surface-initiated polymerization (SIP), e.g., using ATRP (Fig. 1D).

To fabricate the SMM stamp we selected polystyrene microspheres with two different diameters $(5 \mu \mathrm{m}$ and $10 \mu \mathrm{m})$ having a slightly negative surface charge. After the PS microspheres were transferred from an aqueous suspending medium into ethanol, maintaining $\sim 2.0 \%$ solids concentration, they were pipetted onto a slightly tilted silica wafer $(\sim 10 \mathrm{deg})$. The microspheres then selfassembled into a close-packed monolayer by gravitation-induced sedimentation and solvent evaporation., ${ }^{1,21}$ This procedure yielded large areas covered with monolayers of highly ordered, hexagonally packed microspheres with diameters of $5 \mu \mathrm{m}$ (Fig. 2A) and $10 \mu \mathrm{m}$ (Fig. 2B), respectively. Although we observed hexagonal packing over sizeable areas $\left(\sim 0.5 \mathrm{~cm}^{2}\right)$, grain boundaries and packing defects, likely due to surface impurities and the size distribution of the microspheres, did occur. Compared with the fabrication of conventional PDMS $\mu \mathrm{CP}$ stamps, the preparation of our SMM stamps provides an alternative approach that is accessible without specialized equipment. Furthermore, microspheres with a range of chemical functionalities are commercially available and could be selected to further improve self-assembly even over large areas $\left(>10 \mathrm{~cm}^{2}\right)$ in non-polar solvents. ${ }^{22}$ 


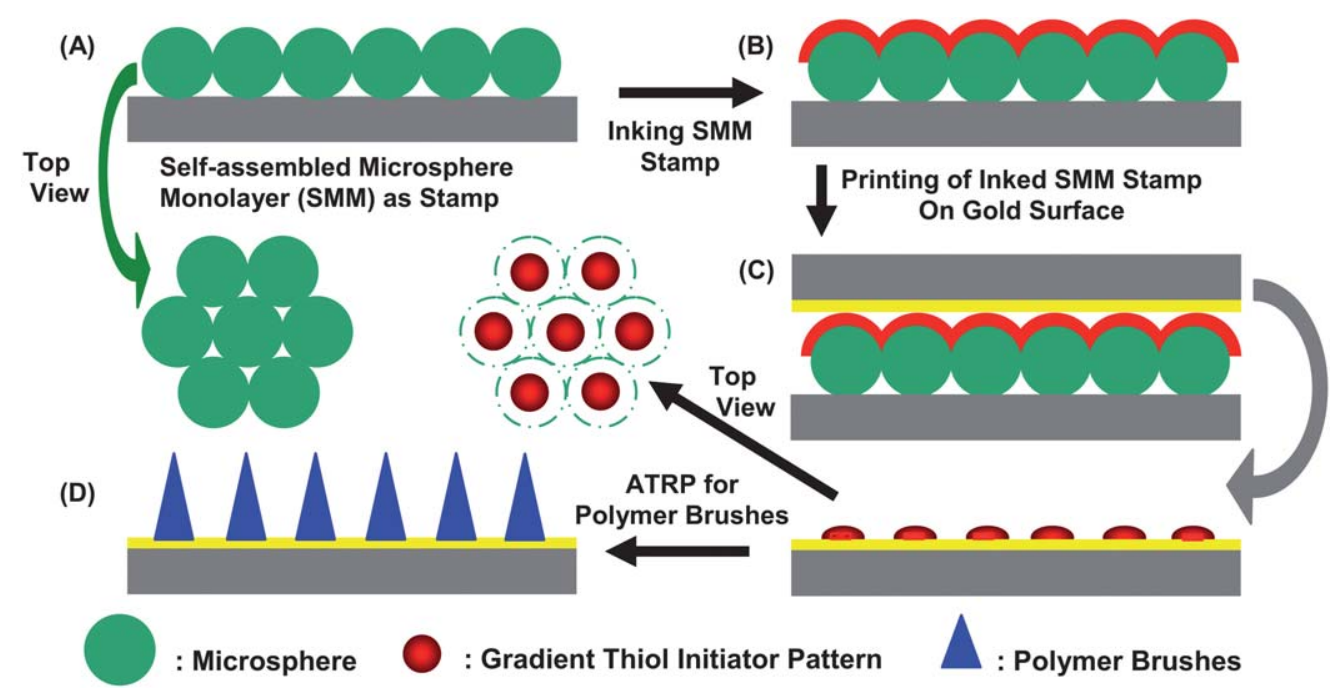

Fig. 1 Schematic illustrations showing the main steps involved in SMM $\mu \mathrm{CP}$ of initiator patterns and their subsequent amplification into patterned polymer brush microstructures by SI-ATRP. (A) SMM stamp prepared by gravitation induced sedimentation in combination with evaporation of the ethanol solvent phase. (B) Dry SMM stamp, inked with a thiol initiator for 3 min and then dried with nitrogen prior to printing. (C) Pattern transfer onto a gold-coated silica wafer using a range of print loads and contact times. (D) Subsequent pattern amplification into cone-shaped polymer brush microstructure by SI-ATRP.

Next, the dry SMM stamp is inked with a thiol initiator by placing a few droplets of ethanolic thiol solution $(2 \mathrm{mM})$ on the SMM stamp surface. After inking, the ethanol is evaporated in a stream of nitrogen to form a dry stamp surface. By bringing the SMM stamp in conformal contact with a gold substrate surface under ambient conditions, the initiator is transferred to the substrate surface. Subsequent amplification of this thiol initiator pattern via SI-ATRP of, e.g., $N$-isopropylacrylamide (NIPAAM), then yields the coneshaped PNIPAAM brush microstructures, shown in Fig. 3A and B. The diameters of the features at half maximal height are $\sim 2.4 \mu \mathrm{m}$ and $\sim 3.8 \mu \mathrm{m}$, for the $5 \mu \mathrm{m}$ and $10 \mu \mathrm{m}$ microsphere SMM stamps, respectively.

Traditional $\mu \mathrm{CP}$ using a PDMS stamp with cylindrical features yields polymer brush microstructures that resemble a truncated cone (Fig. 3C). In contrast, our SMM patterning approach results in conical brush structures (Fig. 3A and B). These differences arise primarily from differences in the stamp features (cylindrical vs. spherical) and their contact with the substrate, as schematically shown in Fig. 3D. For the PDMS stamp with cylindrical features, ink from the recessed areas of the stamp can diffuse onto the gold surface and away from the contact edge of the stamp features, thus effectively increasing the printed footprint area. This can lead to a lower initiator
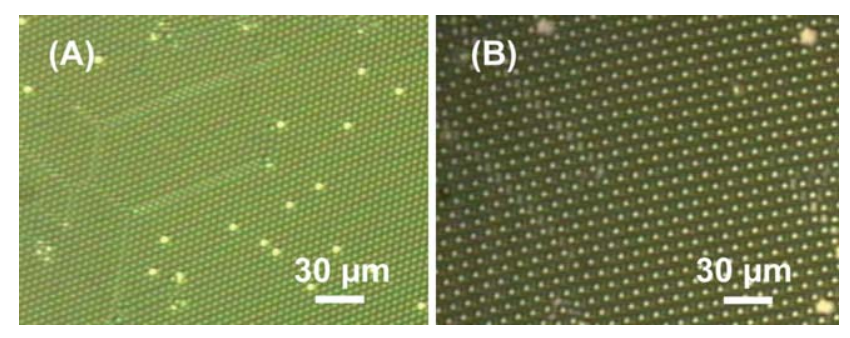

Fig. 2 Optical microscopy images of a typical SMM on a silica wafer (A) using $5 \mu \mathrm{m}$ and (B) $10 \mu \mathrm{m}$ polystyrene microspheres. density in the periphery of the stamp contact area than within it. Lower initiator densities generally cause also lower brush heights under otherwise identical polymerization conditions, ${ }^{23-25}$ which thus explains the truncated cone-shaped polymer brush microstructures shown in Fig. 3C. The ink transfer mechanism for the SMM stamp is similar to that postulated for the PDMS stamp in that thiol initiators diffuse radially outward from the contact area, causing a radially decreasing initiator concentration. In contrast, however, the apparent contact area with the gold surface is likely less than $20 \%$ of the diameter of the microspheres. ${ }^{26}$ The combination of the spherical stamp features with radial thiol diffusion thus explains the coneshaped brush microstructures shown in Fig. 3A and B.

The mechanism of ink transfer observed here is akin to that in edge spreading lithography (ESL) which combines the elements of $\mu \mathrm{CP}$ with dip-pen nanolithography (DPN) ${ }^{27}$ and exploits the spreading of alkanethiol inks by diffusion from a reservoir onto gold substrate surfaces. ${ }^{26}$ For the point contact transfer that occurs with SMM stamps, ink diffusion plays an important role during SMM $\mu \mathrm{CP}$, because it depends primarily on the time in contact as previously shown by McLellan et al. ${ }^{26}$ and by Sharpe et al. ${ }^{28}$ for PDMS stamps. To explore how contact time during SMM $\mu \mathrm{CP}$ can be used to manipulate polymer brush microstructure, we printed ATRP initiators with contact times of $10 \mathrm{~s}, 20 \mathrm{~s}, 40 \mathrm{~s}$, and $60 \mathrm{~s}$. We found (see Fig. S1 in the ESI + ) that with increasing contact time, the initially radially symmetric, conical cross-sectional profiles of the brushes become rougher, with an increasing loss of radial symmetry. We presume that this morphology progression results from unequal diffusion of the thiol ink onto the gold substrate surface, and from an inhomogeneous depletion of ink on the sphere surface.

Our previous research on fabrication of patterned polymer brushes on chemically active surfaces showed that the printed feature size could also be adjusted by affecting the intimacy of contact through the applied load, affecting ink transfer $\mu \mathrm{CP} .{ }^{29} \mathrm{To}$ explore the effect of contact load further, our initiator-inked SMM stamps were brought into contact with the gold substrates for $20 \mathrm{~s}$ at loads of $20 \mathrm{~g}, 50 \mathrm{~g}$, or 

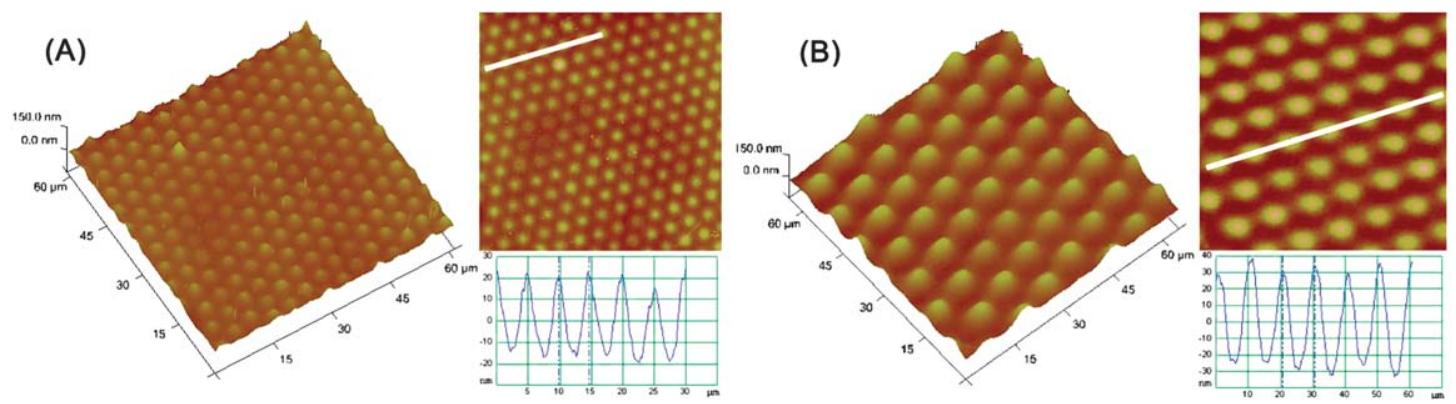

(C)
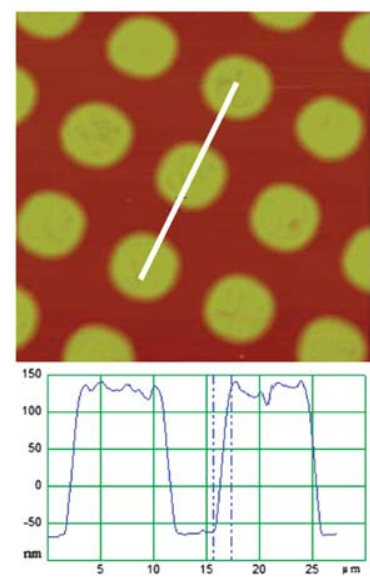

(D)

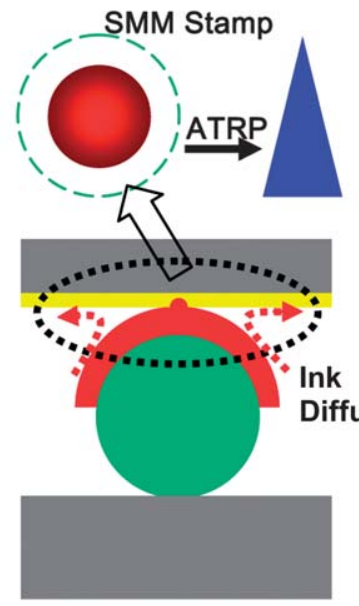

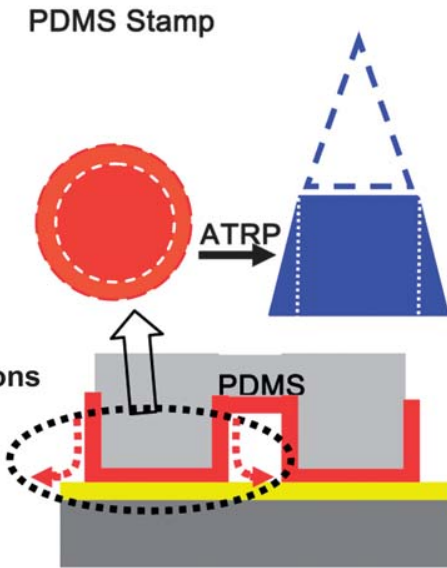

Fig. 3 Contact-mode AFM height images $(60 \mu \mathrm{m} \times 60 \mu \mathrm{m})$ of patterned PNIPPAM brush microstructures imaged at RT in air and the corresponding average height profiles. The polymerization conditions were maintained unchanged in all three examples. Cone-shaped PNIPAAM brush patterns obtained by $\mu \mathrm{CP}$ the initiator with (A) $5 \mu \mathrm{m}$ and (B) $10 \mu \mathrm{m}$ SMM stamps. (C) Truncated cone-shaped PNIPAAM brush pattern obtained by $\mu$ CP the initiator with a conventional PDMS stamp. Print force: $50 \mathrm{~g}$, contact time $20 \mathrm{~s}$. (D) Schematic illustration showing the proposed mechanism of ink diffusion from an inked (left) SMM stamp and (right) PDMS stamp.

$100 \mathrm{~g}$. A plot of the resulting polymer brush feature size vs. print load (Fig. 4) indicates that the feature size depends approximately linearly on the print load (pressure), and has a stronger dependence on load for the larger diameter spheres.

Dry PNIPAAM brushes (prepared by $\mu \mathrm{CP}$ using a $10 \mu \mathrm{m}$ SMM stamp) swell significantly in height (here from $\sim 80 \mathrm{~nm}$ to $\sim 560 \mathrm{~nm}$ ) when exposed to deionized (DI) water $\left(18 \mathrm{M} \Omega \mathrm{cm}^{-1}\right)$ at room temperature. Upon exposure to a $(1: 1, \mathrm{v} / \mathrm{v})$ water/methanol mixture at room temperature, the brushes return to their hydrophobic, collapsed conformation, with a height of $\sim 80 \mathrm{~nm}$. Cyclic exposure to water and a water/methanol mixture showed that this solvent-

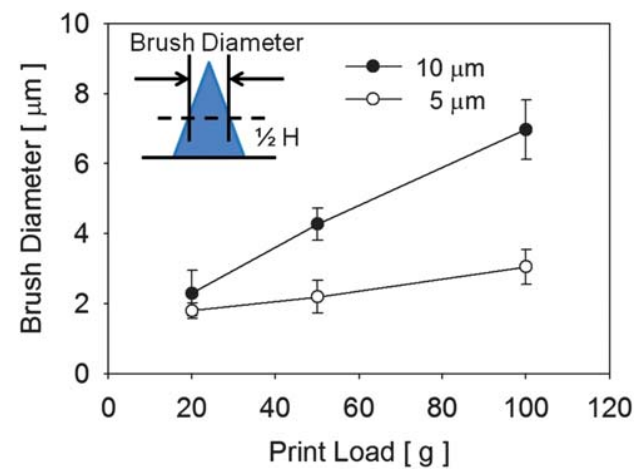

Fig. 4 Plot of feature size versus print force (pressure). The contact time of $20 \mathrm{~s}$ was held constant for all cases. induced phase transition is reversible (Fig. 5), consistent with many previous observations by us and others. ${ }^{25,30}$

In summary, we have exploited self-assembled microsphere monolayers supported on silica wafers as an alternative stamp for $\mu \mathrm{CP}$ and have demonstrated the use of SMM stamps for printing patterned initiator templates that can be subsequently amplified into polymer brushes. Similarly to PDMS $\mu \mathrm{CP}$, SMM $\mu \mathrm{CP}$ provides a promising and simple method for printing microscale SAM patterns over large areas. In our proof of concept demonstration of $\mathrm{SMM} \mu \mathrm{CP}$ for the fabrication of polymer brushes, some variables

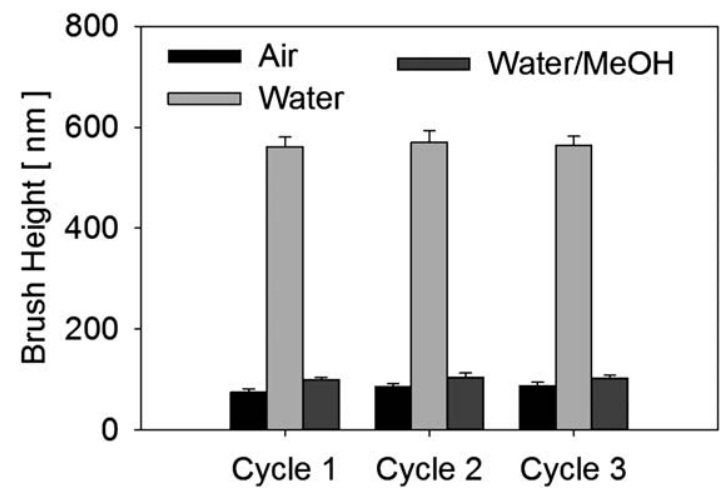

Fig. 5 PNIPAAM brush height plotted as a function of the solvent condition (condition: 0: air; 1: water; 2: mixture of water and methanol, $1: 1, \mathrm{v} / \mathrm{v})$. 
such as the microsphere diameter and the printing conditions (contact time and contact load) were changed, to achieve different polymer brush morphologies. Furthermore, SMM $\mu \mathrm{CP}$ likely provides access to sub-micrometre features due to the point contact ink transfer. A range of commercially available microspheres with different diameters and chemical functionalities could be used to adjust the SMM stamp surface physically (such as stamp feature size) and chemically (such as stamp surface functionality through $-\mathrm{NH} 2,-\mathrm{OH}$, or - $\mathrm{COOH}$ groups). Our work thus provides the point of departure to more systematically establish the interplay of microsphere dimensions, surface chemistry and printing conditions for the transfer of thiols and other inks.

\section{Acknowledgements}

TC and RJ thank the Alexander von Humboldt Foundation for support of TC by an Alexander von Humboldt Research Fellowship. SZ thanks the National Science Foundation for support through grants NSF DMR-0502953 and NSF NIRT CBET-0609265.

\section{References}

1 N. D. Denkov, O. D. Velev, P. A. Kralchevsky, I. B. Ivanov, H. Yoshimura and K. Nagayama, Nature, 1993, 361, 26.

2 M. A. Wood, J. R. Soc. Interface, 2007, 4, 1-17.

3 J. T. Chen, D. Chen and T. P. Russell, Langmuir, 2009, 25, 4331-4335.

4 A. B. Pawar and I. Kretzschmar, Langmuir, 2008, 24, 355-358.

5 V. Kitaev and G. A. Ozin, Adv. Mater., 2003, 15, 75-78.

6 Y. N. Xia, J. Tien, D. Qin and G. M. Whitesides, Langmuir, 1996, 12, 4033-4038.

7 A. Kumar and G. M. Whitesides, Appl. Phys. Lett., 1993, 63, 20022004.

8 Y. N. Xia and G. M. Whitesides, Angew. Chem., Int. Ed., 1998, 37, $551-575$.

9 S. A. Ruiz and C. S. Chen, Soft Matter, 2007, 3, 168-177.
10 T. Kaufmann and B. J. Ravoo, Polym. Chem., 2010, 1, 371-387.

11 A. Perl, D. N. Reinhoudt and J. Huskens, Adv. Mater., 2009, 21, 2257-2268.

12 B. Zhao and W. J. Brittain, Prog. Polym. Sci., 2000, 25, 677-710.

13 W. Senaratne, L. Andruzzi and C. K. Ober, Biomacromolecules, 2005, 6, 2427-2448.

14 T. Chen, R. Ferris, J. M. Zhang, R. Ducker and S. Zauscher, Prog. Polym. Sci., 2010, 35, 94-112.

15 R. Ducker, A. Garcia, J. M. Zhang, T. Chen and S. Zauscher, Soft Matter, 2008, 4, 1774-1786.

16 N. L. Jeon, I. S. Choi, G. M. Whitesides, N. Y. Kim, P. E. Laibinis, Y. Harada, K. R. Finnie, G. S. Girolami and R. G. Nuzzo, Appl. Phys. Lett., 1999, 75, 4201-4203.

17 M. Husemann, D. Mecerreyes, C. J. Hawker, J. L. Hedrick, R. Shah and N. L. Abbott, Angew. Chem., Int. Ed., 1999, 38, 647-649.

18 J. Hyun and A. Chilkoti, Macromolecules, 2001, 34, 5644-5652.

19 P. Xu, H. Uyama, J. E. Whitten, S. Kobayashi and D. L. Kaplan, J. Am. Chem. Soc., 2005, 127, 11745-11753.

20 F. Zhou, Z. J. Zheng, B. Yu, W. M. Liu and W. T. S. Huck, J. Am. Chem. Soc., 2006, 128, 16253-16258.

21 R. M. Erb, N. J. Jenness, R. L. Clark and B. B. Yellen, Adv. Mater., 2009, 21, 4825-4829.

22 M. N. Martin, J. I. Basham, P. Chando and S. K. Eah, Langmuir, 2010, 26, 7410-7417.

23 T. Wu, K. Efimenko and J. Genzer, J. Am. Chem. Soc., 2002, 124, 9394-9395.

24 D. M. Jones, A. A. Brown and W. T. S. Huck, Langmuir, 2002, 18, $1265-1269$.

25 T. Chen, J. M. Zhong, D. P. Chang, A. Carcia and S. Zauscher, Adv. Mater., 2009, 21, 1825-1829.

26 J. M. McLellan, M. Geissler and Y. N. Xia, J. Am. Chem. Soc., 2004, 126, 10830-10831.

27 R. D. Piner, J. Zhu, F. Xu, S. H. Hong and C. A. Mirkin, Science, 1999, 283, 661-663.

28 R. B. A. Sharpe, B. J. F. Titulaer, E. Peeters, D. Burdinski, J. Huskens, H. J. W. Zandvliet, D. N. Reinhoudt and B. Poelsema, Nano Lett., 2006, 6, 1235-1239.

29 T. Chen, D. P. Chang and S. Zauscher, Small, 2010, 6, 1504-1508.

30 F. M. Winnik, H. Ringsdorf and J. Venzmer, Macromolecules, 1990, 23, 2415-2416. 